\title{
Electrorotation of semiconducting microspheres
}

\author{
Laida Rodríguez-Sánchez $\odot$, Antonio Ramos $\odot$, and Pablo García-Sánchez ๑* \\ Departamento Electrónica y Electromagnetismo, Facultad de Física, Universidad de Sevilla, 41012 Sevilla, Spain
}

(Received 22 July 2019; published 25 October 2019)

\begin{abstract}
We study experimentally the electrorotation (ROT) of semiconducting microspheres. $\mathrm{ZnO}$ microspheres obtained by a hydrothermal synthesis method are dispersed in $\mathrm{KCl}$ aqueous solutions and subjected to rotating electric fields. Two ROT peaks are found in experiments: a counterfield peak and a cofield peak at somewhat higher frequencies. These observations are in accordance with recent theoretical predictions for semiconducting spheres. The counterfield rotation is originated by the charging of the electrical double layer at the particleelectrolyte interface, while the cofield rotation is due to the Maxwell-Wagner relaxation. Additionally, we also found that some microspheres in the sample behaved differently and only showed counterfield rotation. We show that the behavior of these particles can be described by the so-called shell model. The microstructure of the microspheres is analyzed with electron microscope techniques and related to the ROT measurements.
\end{abstract}

DOI: 10.1103/PhysRevE.100.042616

\section{INTRODUCTION}

Small particles in suspension can be manipulated by means of electric fields [1,2]. Application of ac voltages to microelectrode arrays is a common way of creating electric fields and acting on particles and/or liquids [3]. Several experimental works have recently shown the capabilities of electric fields for manipulating conducting particles in suspension. For example, semiconductor nanowires are rotated and transported within microchannels [4,5]. Also, electrical properties of semiconductor nanowires can be determined from their orientation rate when subjected to ac fields [6-8]. The rotation velocity of metallic microspheres [9-11] and nanowires [12-14] can be precisely controlled by adjusting the frequency and amplitude of an externally applied rotating electric field. Also, orientation of metal nanowires subjected to ac fields has been extensively reported [15-17]. Self-assembly of metal nanoparticles [18,19] and nanowires [20,21] is achieved on application of ac electric fields. Krupke et al. [22] demonstrated dielectrophoretic separation of semiconducting from conducting nanowires. Electrokinetic transport of metal particles in anisotropic electrolytes (liquid crystals) is also a topic of current research, as recently reviewed in Ref. [23]. The ac field assembly [24,25] and transport [26,27] of Janus metallodielectric spheres are receiving much attention for its potential applications [28]. Moreover, the rotation velocity of semiconducting nanowires subjected to rotating fields can be modulated by light illumination [29,30].

From a theoretical perspective, a number of papers dealing with the modeling of the electrokinetic behavior of metal microparticles have appeared since the late 2000s [10,31-35]. Theory and experiments for metal particles have been compared in several works, as recently reviewed in Ref. [36]. The situation is different for the case of semiconducting particles; scarce fundamental studies have been

\footnotetext{
*pablogarcia@us.es
}

published to date despite the increasing number of papers focusing on applications. We recently compared numerical simulations and experimental data for the rotation and orientation of semiconductor nanowires [37]. Recent analytical models for the electrokinetics of semiconductor spheres predict either cofield or counterfield rotation depending on the frequency of the applied rotating field [38]. The main objective of the present work is to demonstrate that these rotations occur in experiments, together with a close comparison between experimental measurements and theoretical predictions. For that reason, we synthesized $\mathrm{ZnO}$ microspheres and performed a thorough experimental characterization.

The paper is organized as follows: First, and for the sake of completeness, we include a theory section where we reproduce previous theoretical results for the electrorotation (ROT) of a semiconducting sphere immersed in an electrolyte. Later, we describe the particle synthesis and experimental details and show the ROT data that we obtained for the $\mathrm{ZnO}$ microspheres. We compare these data with the theory and draw some conclusions about the properties of the $\mathrm{ZnO}$ microparticles. In particular, we infer that most of the particles are in agreement with the predictions of the theoretical model for homogeneous semiconducting spheres, while a minority of particles are better described by the so-called shell model [1]. We also include electron microscope images of the particles interior that agree with this description.

\section{THEORY}

The theoretical analysis in Ref. [38] deals with the dielectrophoresis and electrorotation of semiconducting spheres immersed in an aqueous electrolyte. In that study we obtained a general expression for the sphere polarizability as a function of electric field frequency. In particular, the expression is valid for arbitrary thickness of the electrical double layer (EDL) induced at the particle-electrolyte interface, i.e., the expression is correct for any relative ratio between the EDL thickness and the sphere radius. The EDL of a semiconductor-electrolyte 


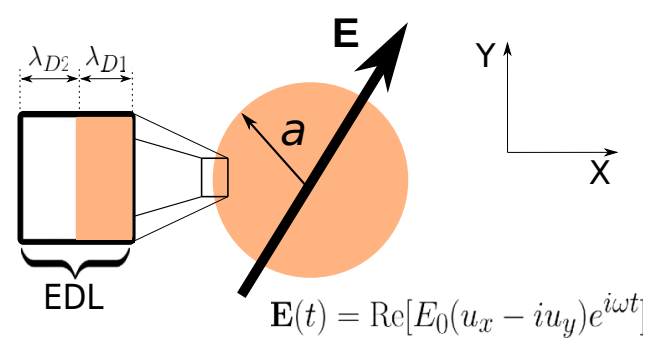

FIG. 1. Schematics of the physical problem. A semiconducting sphere is subjected to a rotating electric field. An EDL is induced at the particle-electrolyte interface.

interface results from the combination of two diffuse layers: one in the semiconducting medium and another in the electrolyte, see Fig. 1. The typical size of a diffuse layer is given by the Debye length, which is around tens of nanometers for both media (see Table I). In the present work, we are interested in comparing theoretical predictions with experimental data for $\mathrm{ZnO}$ microspheres with diameters around $5-15 \mu \mathrm{m}$ and, therefore, the induced EDL is much smaller than the particle radius. The mathematical modeling in this case is simpler since the particle and electrolyte bulks remain electroneutral and the electric potential satisfies Laplace equation, as also shown in Ref. [38]. We include in this section the analysis for the electrorotation of semiconducting spheres in the limit of thin EDL.

Let us consider a semiconducting spheres of radius $a$ immersed in a symmetric aqueous electrolyte such as $\mathrm{KCl}$ in water. The electrolyte conductivity is $\sigma_{1}$ and its dielectric constant $\varepsilon_{1}$. In ROT experiments we assume that the particleelectrolyte system is subjected to an electric field of the form $\mathbf{E}(t)=\operatorname{Re}\left[E_{0}\left(\boldsymbol{u}_{x}-i \boldsymbol{u}_{y}\right) e^{i \omega t}\right]$, where $i$ is the imaginary unit and $\operatorname{Re}[\cdots]$ stands for the real part of $[\cdots]$, see Fig. 1 . This electric field corresponds to a homogeneous field of constant magnitude and rotating counterclockwise with angular frequency $\omega$ within the xy plane. The electric field induces an electric dipole on the particle $\mathbf{p}$. Using phasors, the induced dipole is written as $\mathbf{p}=\operatorname{Re}\left[\tilde{\mathbf{p}} e^{i \omega t}\right]$, where $\tilde{\mathbf{p}}$ is the dipole phasor and can be written as a function of the particle polarizability as $\tilde{\mathbf{p}}=\tilde{\alpha} \tilde{\mathbf{E}}$. Correspondingly, $\tilde{\mathbf{E}}$ is the electric field phasor. The particle polarizability is usually expressed as a function of the Clausius-Mossotti factor, $\tilde{K}(\omega)$, according to $\tilde{\alpha}=4 \pi \varepsilon_{1} a^{3} \tilde{K}(\omega)$. The time-averaged electrical torque on the system is $\boldsymbol{\tau}(\mathrm{ROT})=(1 / 2) \operatorname{Re}\left[\tilde{\mathbf{p}} \times \tilde{\mathbf{E}}^{*}\right]$ and can be written as follows:

$$
\boldsymbol{\tau}(\mathrm{ROT})=-4 \pi \varepsilon_{1} a^{3} \operatorname{Im}[\tilde{K}(\omega)] E_{0}^{2} \mathbf{u}_{z},
$$

where $\operatorname{Im}[\cdots]$ stands for the imaginary part of $[\cdots]$.

TABLE I. Physical properties for the electrolyte and the semiconductor sphere in Fig. 2. Data for $\mathrm{ZnO}$ are within the ranges reported in Refs. [42,43].

\begin{tabular}{lccc}
\hline \hline & $\lambda_{D}$ & $\sigma$ & $\varepsilon / \varepsilon_{0}$ \\
\hline Semiconductor $(\mathrm{ZnO})$ & $50 \mathrm{~nm}$ & $263 \mathrm{mS} / \mathrm{m}$ & 3.9 \\
Electrolyte $(\mathrm{KCl})$ & $30 \mathrm{~nm}$ & $1.5 \mathrm{mS} / \mathrm{m}$ & 80 \\
Electrolyte $(\mathrm{KCl})$ & $9.5 \mathrm{~nm}$ & $15 \mathrm{mS} / \mathrm{m}$ & 80 \\
\hline \hline
\end{tabular}

For negligible particle inertia, the electrical torque is balanced by the viscous torque that the fluid exerts on the particle. In experiments, the spheres rest on a substrate and, therefore, we use the expression for the viscous torque on a spherical particle resting on a wall that rotates with angular velocity $\Omega$ around an axis perpendicular to the wall [39]: $\boldsymbol{\tau}_{\text {viscous }}=-8 \pi \eta a^{3} \zeta(3) \boldsymbol{\Omega}$, where $\eta$ is the fluid viscosity and $\zeta(3) \approx 1.20206$. Solving for $\boldsymbol{\Omega}$ :

$$
\boldsymbol{\Omega}=-\frac{\varepsilon_{1}}{2 \zeta(3) \eta} \operatorname{Im}[\tilde{K}(\omega)] E_{0}^{2} \mathbf{u}_{z} .
$$

The effect of the wall on the electric field and torque is much smaller [38] and we neglect it in the present analysis.

\section{Particle polarizability}

According to the analysis above, we have to find the Clausius-Mossotti factor of the system in Fig. 1. To this end, we assume that the particle-electrolyte system is subjected to a homogeneous ac electric field given by $\mathbf{E}=\operatorname{Re}\left[E_{0} \mathbf{u}_{x} e^{i \omega t}\right]$ and, therefore, the potential far from the particle can be written in spherical coordinates as $\phi(r \rightarrow \infty)=-\operatorname{Re}\left[E_{0} r \cos \theta e^{i \omega t}\right]$. As mentioned above, we assume that the EDL at the particleelectrolyte interface is much smaller than the sphere radius. Therefore, the particle and liquid bulks remain electroneutral after application of the electric field and the electric potential satisfies Laplace equation $\nabla^{2} \phi_{1}=0$ and $\nabla^{2} \phi_{2}=0$, where the subscript 1 refers to the particle (medium 1 ) and subscript 2 to the electrolyte (medium 2). The following boundary condition at the particle surface accounts for the voltage drop across the EDL at the electrolyte-particle interface [40]:

$$
\left.\left(\phi_{2}-\phi_{1}\right)\right|_{a}=\left.\sigma_{2} \frac{\partial \phi_{2}}{\partial r}\right|_{a} \tilde{Z}_{2}+\left.\sigma_{1} \frac{\partial \phi_{1}}{\partial r}\right|_{a} \tilde{Z}_{1}
$$

where $\sigma_{j}$ and $\varepsilon_{j}$ are, respectively, the conductivity and permittivity of medium $j(j=1,2) . \tilde{Z}_{j}$ stands for the surface impedance due to the thin diffuse layer in medium $j$ :

$$
\tilde{Z}_{j}=\frac{\lambda_{D j}}{i \omega \varepsilon_{j} \sqrt{1+i \omega \varepsilon_{j} / \sigma_{j}}} .
$$

Equation (3) was found by Zhao et al. [41] when studying the ac electrosmostic flow that might appear at electrolytesemiconductor interfaces.

Additionally, the conservation of the total current (ohmic plus displacement current) across the particle-electrolyte interface results in another boundary condition:

$$
\left.\left(\sigma_{2}+i \omega \varepsilon_{2}\right) \frac{\partial \phi_{2}}{\partial r}\right|_{a}=\left.\left(\sigma_{1}+i \omega \varepsilon_{1}\right) \frac{\partial \phi_{1}}{\partial r}\right|_{a} .
$$

Equation (5) holds as long as tangential currents at the particle surface are negligible.

The solutions to the electric potential in the electrolyte and particle can be written as:

$$
\begin{gathered}
\phi_{1}=-E_{0} r \cos \theta+\frac{A}{r^{2}} \cos \theta, \\
\phi_{2}=B r \cos \theta,
\end{gathered}
$$

where $A$ and $B$ are integration constants to be determined after applying boundary conditions (3) and (5). Taking into account 


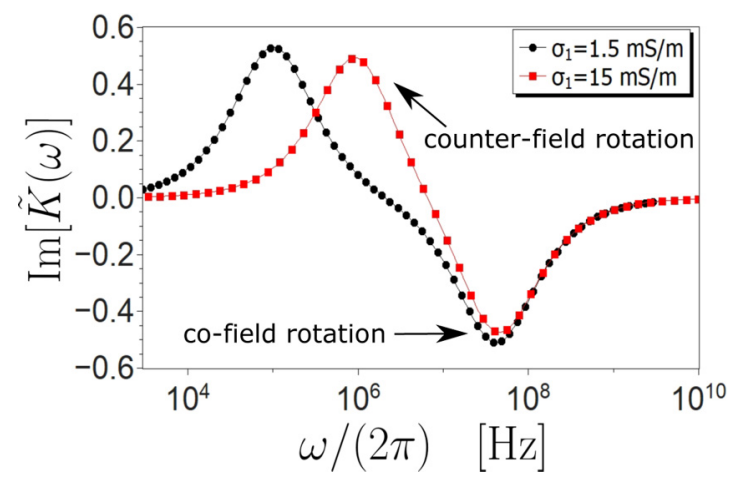

FIG. 2. Imaginary part of the Clausius-Mossotti factor versus signal frequency for a $\mathrm{ZnO}$ microsphere immersed in a $\mathrm{KCl}$ solution. Data for material properties are shown in Table I and the microsphere is $10-\mu \mathrm{m}$ diameter.

that $A$ and the Clausius-Mossotti factor are related by $\tilde{K}=$ $A /\left(a^{3} E_{0}\right)$, we readily obtain:

$$
\tilde{K}(\omega)=\frac{1-H(\omega)}{1+2 H(\omega)},
$$

where the function $H(\omega)$ is defined as:

$$
H(\omega)=\frac{\sigma_{1}+i \omega \varepsilon_{1}}{\sigma_{2}+i \omega \varepsilon_{2}}\left(1+\frac{\sigma_{2} \tilde{Z}_{2}}{a}\right)+\left(\frac{\sigma_{1} \tilde{Z}_{1}}{a}\right) .
$$

For high frequencies, $H(\omega)$ tends to the ratio of liquid to solid permittivity. In this limit, Eq. (8) becomes the well-known Clausius-Mossotti factor for a dielectric sphere in a dielectric medium [1].

Figure 2 shows the prediction of the model for a $10-\mu \mathrm{m}$ semiconductor sphere with the properties shown in Table I and for two typical electrolyte conductivities $\sigma_{1}=1.5,15 \mathrm{mS} / \mathrm{m}$. The imaginary part of the Clausius-Mossotti factor shows two different peaks: a positive peak at low frequencies that indicates a counterfield rotation according to Eq. (2), and a negative peak at higher frequencies that corresponds to cofield rotation. As described in our previous work [38], the counterfield peak is related to the charging of the EDL capacitance through resistive media at the particle-electrolyte interface. On the other hand, the cofield rotation is originated by the Maxwell-Wagner relaxation arising from the polarization of the interface between two media with different conductivities and/or permittivities [1].

The electric field acting on the induced EDL can also give rise to electro-osmotic flows as the induced-charge electrosmotic (ICEO) flows reported for metal particles [44]. However, under the approximation of a thin EDL, the ICEO flow induced by a rotating field on a sphere does not possess any rotating component and, therefore, it does not induce particle rotation [21]. In other terms, Eq. (2) is not affected by ICEO flows.

Furthermore, the theoretical model above does not consider the presence of an intrinsic surface charge or intrinsic EDL at the particle-electrolyte interface, which might be present as usually happens on metal oxide surfaces in contact with water. It is well known that this intrinsic surface charge can give rise to surface conductance that leads to another relaxation phenomenon, commonly known as $\alpha$ relaxation [45]. The typical (a)

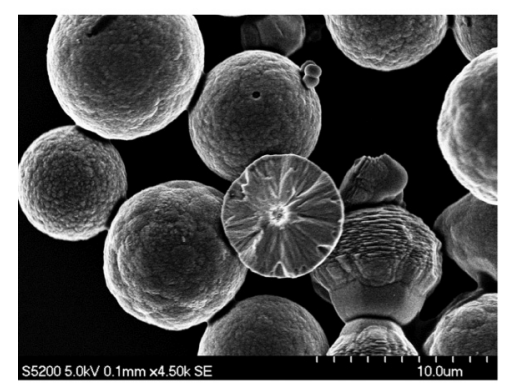

(b)

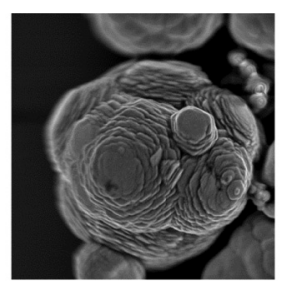

(c)

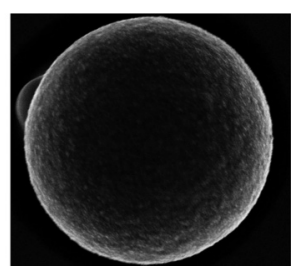

FIG. 3. (a) SEM image of $\mathrm{ZnO}$ particles obtained by a hydrothermal synthesis method. (b) A representative rough $\mathrm{ZnO}$ sphere. (c) A typical smooth $\mathrm{ZnO}$ sphere.

frequency for this relaxation is $f=(1 / 2 \pi)\left(D / a^{2}\right)$, where $D$ is the ion diffusion constant. Thus, for a particle with $a \approx 5 \mu \mathrm{m}$ in $\mathrm{KCl}$ results $f \approx 10 \mathrm{~Hz}$, well below the frequency range in our study. Additionally, the action of the rotating field on this intrinsic EDL can induce a nonhomogeneous $\zeta$ potential that gives rise to electro-osmotic flow around the particle, leading to cofield particle rotation [46]. This cofield rotation could occur for our semiconducting spheres but also for frequencies of the $\alpha$ relaxation.

\section{EXPERIMENTAL METHODS AND RESULTS}

Zinc-oxide particles were obtained by following a hydrothermal synthesis method described in Ref. [47]: $1 \mathrm{mmol}$ zinc nitrate hexahydrate $\left[\mathrm{Zn}\left(\mathrm{NO}_{3}\right)_{2} \cdot 6 \mathrm{H}_{2} \mathrm{O}\right]$ powder was mixed with $16 \mathrm{ml}$ of absolute ethanol. Later $0.5 \mathrm{mmol}$ anhydrous ethylenediamine was added into the solution and stirred for about $10 \mathrm{~min}$. The solution was transferred into a $20-\mathrm{ml}$ Teflon-lined stainless steel autoclave and maintained at $180^{\circ} \mathrm{C}$ for around $2 \mathrm{~h}$. Figure 3 shows Scanning Electron Microscope (SEM) images of $\mathrm{ZnO}$ particles obtained by this method. Most particles have a nearly spherical shape, with diameters ranging between 5 and $15 \mu \mathrm{m}$. Curiously, two particles populations can be clearly identified: $\mathrm{ZnO}$ quasispherical particles with a rough surface, see Fig. 3(b), and ZnO spherical particles with a smooth surface, see Fig. 3(c).

Electrorotation experiments are visualized with an inverted optical microscope equipped with a $40 \times$ objective. This allows us to distinguish between rough and smooth spheres, but for a clear measurement of the rotation velocity, it is convenient to decorate the particle surface before the experiments. To this end, we redispersed the $\mathrm{ZnO}$ particles in a high-conductivity $\mathrm{KCl}$ water solution containing small fluorescent polystyrene beads (500-nm diameter). Under these conditions, some fluorescent beads irreversibly attached to the particle surface [Fig. 4(b)]. Subsequently, the samples were redispersed in $\mathrm{KCl}$ water solutions with a lower conductivity $(1.5,5$, and $15 \mathrm{mS} / \mathrm{m})$ and a small sample volume is pipetted on top of an array of four coplanar electrodes. The electrodes have a circular shape and they are made of platinum on a 
(a)

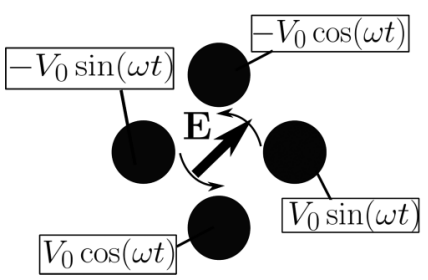

(b)

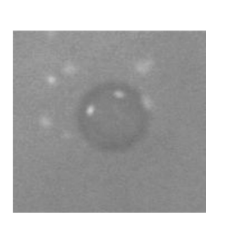

FIG. 4. (a) Schematics of the electrode signals and the resulting counterclockwise rotating field. (b) $\mathrm{ZnO}$ sphere decorated with 500-nm fluorescent beads.

glass substrate. The distance between opposite electrodes is $1 \mathrm{~mm}$. This electrode array is employed to generate a rotating electric field, as shown schematically in Fig. 4(a). For that reason, each electrode is subjected to a sinusoidal voltage with peak-to-peak amplitude of $20 \mathrm{~V}$ and frequencies in the range $1 \mathrm{kHz}-7 \mathrm{MHz}$. The maximum frequency is limited by

the generator specifications. In order to achieve a rotating field, we impose a phase lag of $90^{\circ}$ between the ac signals of neighboring electrodes. Videos of the rotating particles are captured with a digital camera attached to the microscope for later analysis. From these videos, we measured the rotation period of the $\mathrm{ZnO}$ particles as the average time per rotation after five turns at least.

\section{A. Electrorotation data}

Figure 5 shows the measurements of the rotation velocity of $\mathrm{ZnO}$ particles (in units of revolutions per second) as a function of the frequency of the rotating electric field. The left column includes data for rough spheres at three different electrolyte conductivity: $1.5,5$, and $15 \mathrm{mS} / \mathrm{m}$. Data for smooth spheres and the same electrolyte conductivities are included in the right column. Positive values of the rotation velocity indicate cofield rotation, while negative values correspond to counterfield rotation. Remarkably, rough spheres show both
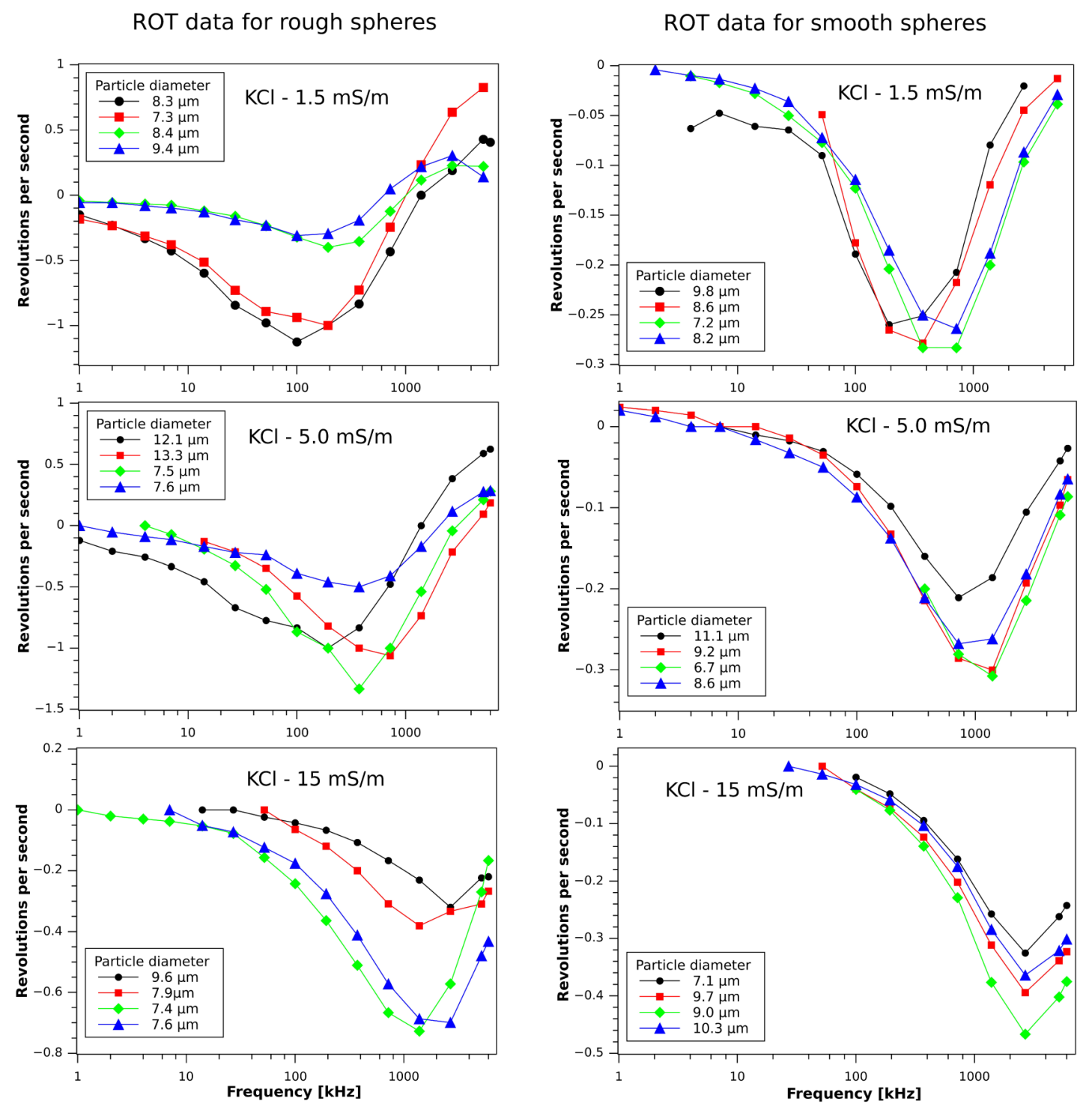

FIG. 5. Rotation velocity of $\mathrm{ZnO}$ spheres as a function of the frequency of the rotating applied field. Left column shows data for rough spheres dispersed in $\mathrm{KCl}$ aqueous solutions with three different conductivities. Likewise, the right column shows data for smooth spheres. 


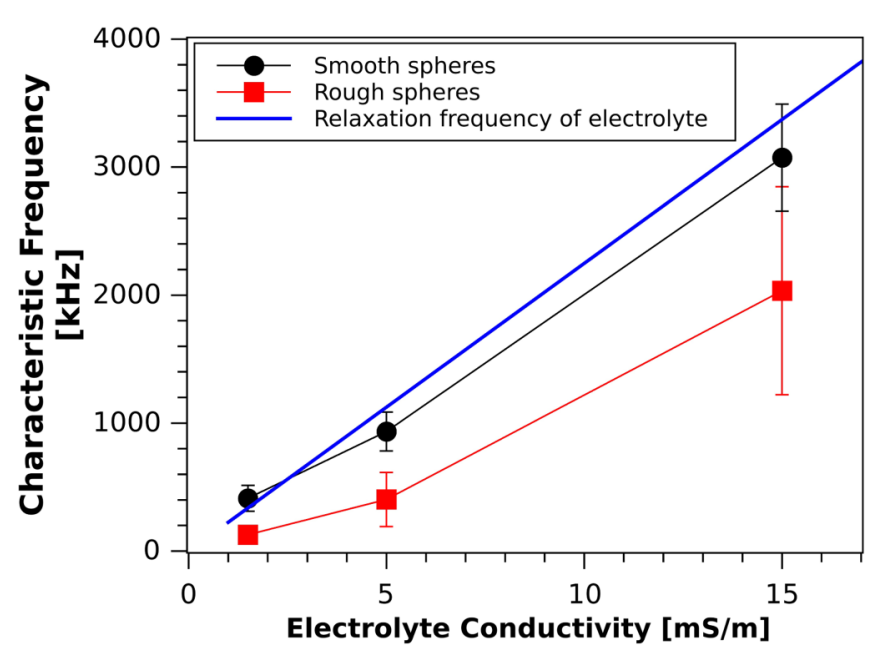

FIG. 6. Characteristic frequency for maximum counterfield rotation as a function of the electrolyte conductivity. The plot also shows the charge relaxation frequency for the electrolyte bulk, $f_{1}=$ $\sigma_{1} /\left(2 \pi \varepsilon_{1}\right)$.

counter- and cofield rotation, as theoretically predicted for homogeneous semiconducting spheres. The signal frequency for maximum rotation velocity increases with electrolyte conductivity; this trend is found for both the counter- and the cofield peaks, and it is also in agreement with the theoretical expectation (Fig. 2). The limited frequency range of the signal generator precluded the observation of cofield rotation for rough spheres in $15 \mathrm{mS} / \mathrm{m} \mathrm{KCl}$. Presumably, the cofield rotation of smooth spheres was not observed for the same reason, while their counterfield rotation peak was clearly measured for all electrolyte conductivities.

Figure 6 shows the characteristic frequency for maximum counterfield rotation as a function of the electrolyte conductivity. This characteristic frequency is obtained by fitting the ROT data of each particle to a lorentzian curve. Data shown in Fig. 6 correspond to the average of the characteristic frequencies of several spheres (around 10) for a given electrolyte conductivity. Error bars are the standard deviation of the characteristic frequencies. Note that we are averaging over particles with different diameters (around 30\% difference). However, the main reason for the variability in ROT spectra is not the size dispersion but the particle conductivity, as shown below. Characteristic frequencies for smooth spheres are higher than for rough spheres. Figure 6 also shows the charge relaxation frequency for the electrolyte bulk, $f_{1}=\sigma_{1} /\left(2 \pi \varepsilon_{1}\right)$. Importantly, the characteristic frequencies for smooth spheres are very close to $f_{1}$. As shown in the next section, this indicates that smooth spheres do not behave as expected for homogeneous semiconducting spheres.

\section{B. Comparison with theory}

Before comparing theory and experiments, we have to take into account that the applied rotating field is not homogeneous within the observation region. For this reason, we use the average value of the electrical torque within the field of view. This was calculated in Ref. [13] for the same electrode geometry; the theoretical value for ROT velocity can be calculated from expression (2) and using that $E_{0}^{2}=10^{8} \mathrm{~V}^{2} / \mathrm{m}^{2}$.
ROT data for rough spheres are fitted to the theoretical model in Sec. 2. The relative size of the roughness is much greater than the EDL thickness, which is around $100 \mathrm{~nm}$ or below. Thus, the theoretical approximation for a thin EDL remains valid. We expect this roughness to have a small effect on the ROT spectra. For example, experiments with gold-coated microspheres [10] show that the counter-rotation peak occurs at frequencies somewhat lower than in theory. The particles in these experiments consisted in latex spheres coated with colloidal gold and the lower frequencies were attributed to the rough surface resulting from the coating procedure. Since the electrolyte properties are known (Table 1 and $\eta=10^{-3} \mathrm{Pas}$ ), the only fitting parameter is the $\mathrm{ZnO}$ conductivity. Note that the Debye length for $\mathrm{ZnO}$ is not a fitting parameter but it is computed for each $\mathrm{ZnO}$ conductivity according to the scaling $\lambda_{\mathrm{D} 2} \propto 1 / \sqrt{\sigma_{2}}$. Figure 7 (a) shows the theoretical curves for $\sigma_{1}=5 \mathrm{mS} / \mathrm{m}$ and two $\mathrm{ZnO}$ conductivities, $\sigma_{2}=70$ and $1 \mathrm{mS} / \mathrm{m}$. The sphere diameter for the theoretical calculations corresponds to the average of experimental diameters in Fig. 5. The theoretical curves enclose most of the experimental data for rough spheres, but more importantly, theory and experiment show the same trend: When the frequency for maximum counterfield rotation is higher, the rotation velocity is smaller and the cofield rotation appears at lower frequencies (Figs. 5 and 7). Thus, we attribute the variability in the ROT velocity of rough spheres to the differences in conductivity: $\mathrm{ZnO}$ spheres with a smaller rotation velocity are less conducting than faster microspheres. The range of $\mathrm{ZnO}$ conductivities expands almost two orders of magnitude. According to Janotti et al. [42] the control of $\mathrm{ZnO}$ conductivity (and, as consequence, its Debye length) is a major issue. For example, electrical properties of $\mathrm{ZnO}$ are very sensitive to surface modifications. Also, relatively small concentrations of native point defects and impurities significantly affect the electrical and optical properties of semiconductors. The conduction mechanism in $\mathrm{ZnO}$ is a topic of research itself [48] which is beyond the scope of the present work.

We have also tried to fit the theoretical model to the experimental data for smooth $\mathrm{ZnO}$ spheres. Since the ROT peak for these spheres is found at higher frequencies, one would expect that the data could be fitted with even smaller values for the $\mathrm{ZnO}$ conductivity, $\sigma_{2}$. However, as $\sigma_{2}$ decreases (and $\lambda_{\mathrm{D} 2}$ correspondingly increases), the theoretical ROT velocities also decrease and it turns out that it is not possible to simultaneously fit the signal frequency and amplitude of maximum ROT speed. This is an indication that our model for homogeneous semiconductor spheres is not suitable for describing the electrokinetic behavior of the smooth spheres. As mentioned above, the characteristic frequency for smooth spheres approaches the charge relaxation frequency of the electrolyte (Fig. 6). This suggests us the possibility of modeling the sphere behavior by using a shell model [1] that consists in a dielectric spherical shell surrounding a conducting core. Figure 7(b) shows the predictions for an electrolyte conductivity of $5 \mathrm{mS} / \mathrm{m}$ and a shell model consisting in a $2-\mu \mathrm{m}$-thick dielectric shell with the same permittivity as $\mathrm{ZnO}$ $\left(\varepsilon_{2} / \varepsilon_{0}=3.9\right)$ surrounding a $\mathrm{ZnO}$ core with the same electrical properties than used for the rough spheres. The introduction of this insulating layer does not imply that the particle radius increases: rough and smooth spheres have the same sizes. The 
(a)

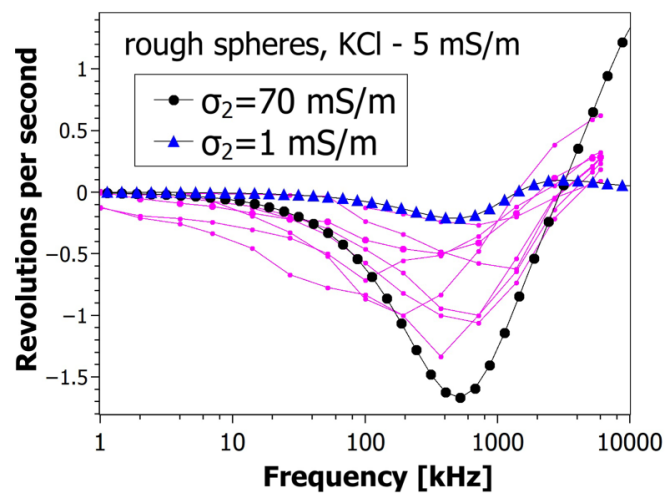

(b)

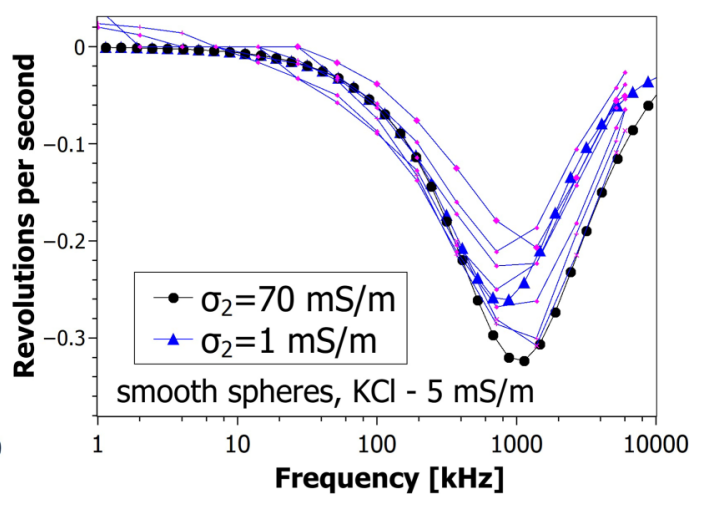

FIG. 7. (a) Comparison between experimental data for rough spheres with $\sigma_{1}=5 \mathrm{mS} / \mathrm{m}$ and the theoretical model for two particle conductivities: $\sigma_{2}=70$ and $1 \mathrm{mS} / \mathrm{m}$. (b) Experimental data for smooth spheres with $\sigma_{1}=5 \mathrm{mS} / \mathrm{m}$ and the theoretical prediction of the shell model

shell model works because the introduction of an insulating layer acts as a capacitance in series with the capacitances due to the diffuse layers in the electrolyte and semiconducting core. The capacitance of this thick insulating layer is much smaller and, since it is in series with the diffuse layers, it dominates the overall capacitance of the interface. Thus, the typical resistor-capacitor (RC) circuit time is now determined by the insulating layer. As the RC time diminishes, the corresponding typical frequency increases, approaching the electrolyte charge relaxation frequency. The presence of a conducting core ensures that the magnitude of the induced dipole in the particle remains relatively big, as is the magnitude of the ROT velocity. In the next section we use electron microscope techniques to investigate whether this shell model represents some real structure of the smooth spheres.

\section{Electron microscope images of the particles}

The surface topography of the $\mathrm{ZnO}$ spheres was studied with an SEM microscope, as described in Sec. 3, see Fig. 3. The ROT data for smooth spheres are in good agreement with (a)

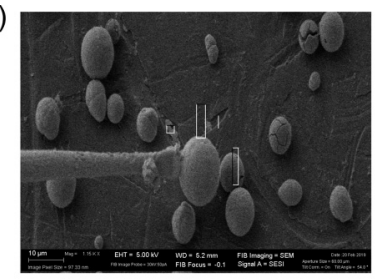

(c)

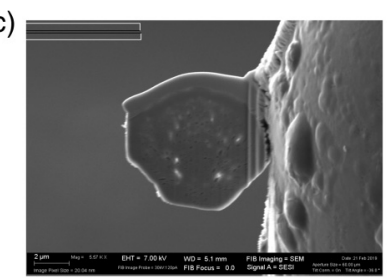

(b)

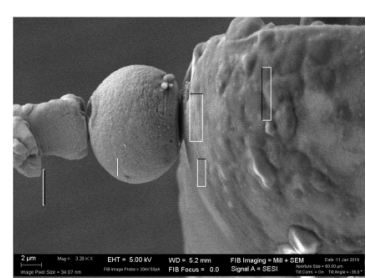

(d)

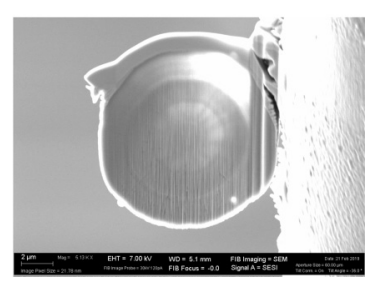

FIG. 8. (a)A smooth particle is picked by using the micromanipulator. (b) A particle is welded to a metal holder before using the ion beam. (c) Cross-section image of a rough particle. (d) Cross-section image of a smooth particle. the predictions of a particle model consisting in a dielectric shell surrounding a conducting particle. This result motivated us to analyze the internal structure of the spheres. For this purpose, we made use of an electron microscope (Zeiss Auriga) equipped with an ion beam that enables the slicing of the particles. This microscope also includes a micromanipulator which allowed us to select one specific particle out of the sample. For example, Fig. 8(a) shows a smooth particle picked by the micromanipulator. This sphere is later welded to a metal holder, see Fig. 8(b). Subsequently, a gallium ion beam is used for slicing the particle. Figure 8(c) shows an SEM image of the interior of a rough particle and Fig. 8(d) is an image of a smooth particle. Rough spheres display interstices but look homogeneous on a large scale. Smooth spheres are different, and a concentric layering is evident from the SEM images.

Previous analyses of $\mathrm{ZnO}$ particles obtained by hydrothermal methods demonstrate that the particles can show different crystalline and/or amorphous structures [49,50]. For example, Inguva et al. [51] used high-resolution Transmission Electron Microscopy (HR-TEM) to show that their $\mathrm{ZnO}$ nanowires consist in a crystalline core with an amorphous shell. We have also taken our samples to an HR-TEM (FEI Talos). Beforehand, the particles were further sliced until reaching a thin layer. The results show that rough $\mathrm{ZnO}$ spheres are crystalline with a wurtzite-type structure, while the outer layer of the smooth spheres consists of small islands of crystalline material immersed in an amorphous $\mathrm{ZnO}$ shell. We thus believe that this amorphous shell behaves as the insulating layer that determines the ROT behavior of smooth particles.

\section{CONCLUSIONS}

We have measured the ROT spectra of semiconducting microspheres for the first time. $\mathrm{ZnO}$ particles were obtained by a hydrothermal synthesis method and dispersed in $\mathrm{KCl}$ aqueous solutions. Most of the semiconducting microspheres show two ROT peaks: one counterfield and a cofield peak at somewhat higher frequencies. This behavior is in accordance with the theoretical model for homogeneous semiconducting spheres. The counterfield rotation is originated by the charging of the electrical double layer induced at the particle-electrolyte 
interface, while the cofield rotation is due to the MaxwellWagner relaxation. Experimental data are satisfactorily fitted to the theoretical predictions by using only one fitting parameter, the particle electrical conductivity.

Additionally, we have also found that some of the $\mathrm{ZnO}$ microspheres have a smoother surface and show a different behavior: They only have one counterfield rotation peak within the limited frequency range of the rotating electric field (up to $7 \mathrm{MHz}$ ). Experimental data for the smooth spheres cannot be fitted to the predictions of the theoretical model for homogeneous semiconductors. Instead, we have used the socalled shell model consisting in a $2-\mu \mathrm{m}$-thick dielectric layer that surrounds a conducting core with the same properties as the rough particles. Electron microscope results show that rough spheres are homogeneous and possess a crystalline structure [Fig. 8(c)], while smoother spheres show a structure of concentric layers [Fig. 8(d)], supporting the hypothesis that the latter particles are not homogeneous but bear an insulating shell that determines their ROT behavior.

\section{ACKNOWLEDGMENTS}

L.R.-S. acknowledges Junta de Andalucía Contract No. PEJUS-2. The authors acknowledge Francisco Varela from CITIUS (Universidad de Sevilla) for his technical work with the electron microscopes. We acknowledge financial support from Spanish Government Ministry MICINN under Contract No. PGC2018-099217-B-I00.
[1] T. B. Jones, Electromechanics of Particles (Cambridge University Press, Cambridge, 1995).

[2] A. Ramos, Electrokinetics and Electrohydrodynamics in Microsystems (Springer Science \& Business Media, New York, 2011), Vol. 530.

[3] H. Morgan and N. G. Green, AC Electrokinetics: Colloids and Nanoparticles (Research Studies Press Ltd., Baldock, Hertfordshire, 2003).

[4] K. Yu, J. Yi, and J. W. Shan, Lab Chip 18, 1494 (2018).

[5] D. Fan, F. Q. Zhu, X. Xu, R. C. Cammarata, and C. Chien, Proc. Natl. Acad. Sci. USA 109, 9309 (2012).

[6] W. Yuan, G. Tutuncuoglu, A. Mohabir, L. Liu, L. C. Feldman, M. A. Filler, and J. W. Shan, Small 15, 1805140 (2019).

[7] C. Akin, L. C. Feldman, C. Durand, S. M. Hus, A.-P. Li, H. Y. Hui, M. A. Filler, J. Yi, and J. W. Shan, Lab Chip 16, 2126 (2016).

[8] C. Akin, J. Yi, L. C. Feldman, C. Durand, S. M. Hus, A.-P. Li, M. A. Filler, and J. W. Shan, ACS Nano 9, 5405 (2015).

[9] Y. Ren, D. Morganti, H. Y. Jiang, A. Ramos, and H. Morgan, Langmuir 27, 2128 (2011).

[10] P. García-Sánchez, Y. Ren, J. J. Arcenegui, H. Morgan, and A. Ramos, Langmuir 28, 13861 (2012).

[11] J. J. Arcenegui, P. García-Sánchez, H. Morgan, and A. Ramos, Electrophoresis 34, 979 (2013).

[12] D. L. Fan, F. Q. Zhu, R. C. Cammarata, and C. L. Chien, Phys. Rev. Lett. 94, 247208 (2005).

[13] J. J. Arcenegui, P. García-Sánchez, H. Morgan, and A. Ramos, Phys. Rev. E 88, 033025 (2013).

[14] J. J. Arcenegui, P. García-Sánchez, H. Morgan, and A. Ramos, Phys. Rev. E 88, 063018 (2013).

[15] D. Saintillan, E. Darvel, and E. Shaqfeh, J. Fluid Mech. 563, 223 (2006).

[16] K. A. Rose, J. A. Meier, G. M. Dougherty, and J. G. Santiago, Phys. Rev. E 75, 011503 (2007).

[17] J. J. Arcenegui, P. García-Sánchez, H. Morgan, and A. Ramos, Phys. Rev. E 89, 062306 (2014).

[18] K. D. Hermanson, S. O. Lumsdon, J. P. Williams, E. W. Kaler, and O. D. Velev, Science 294, 1082 (2001).

[19] B. Gierhart, D. Howitt, S. Chen, R. Smith, and S. Collins, Langmuir 23, 12450 (2007).
[20] P. A. Smith, C. D. Nordquist, T. N. Jackson, T. S. Mayer, B. R. Martin, J. Mbindyo, and T. E. Mallouk, Appl. Phys. Lett. 77, 1399 (2000).

[21] P. García-Sánchez, J. J. Arcenegui, H. Morgan, and A. Ramos, Appl. Phys. Lett. 106, 023110 (2015).

[22] R. Krupke, F. Hennrich, H. v. Löhneysen, and M. M. Kappes, Science 301, 344 (2003).

[23] C. Peng and O. D. Lavrentovich, Micromachines 10, 45 (2019).

[24] S. Gangwal, A. Pawar, I. Kretzschmar, and O. D. Velev, Soft Matter 6, 1413 (2010).

[25] S. Gangwal, O. J. Cayre, and O. D. Velev, Langmuir 24, 13312 (2008).

[26] S. Gangwal, O. J. Cayre, M. Z. Bazant, and O. D. Velev, Phys. Rev. Lett. 100, 058302 (2008).

[27] A. Boymelgreen, G. Yossifon, and T. Miloh, Langmuir 32, 9540 (2016).

[28] A. M. Boymelgreen, T. Balli, T. Miloh, and G. Yossifon, Nat. Commun. 9, 760 (2018).

[29] F. Cao, D. Yu, Y. Gu, J. Chen, and H. Zeng, Nanoscale 11, 3117 (2019).

[30] Z. Liang and D. Fan, Sci. Adv. 4, eaau0981 (2018).

[31] T. Miloh, Phys. Fluids 21, 072002 (2009).

[32] T. Miloh, Phys. Fluids 20, 063303 (2008).

[33] T. Miloh and B. W. Goldstein, Phys. Fluids 27, 022003 (2015).

[34] P. García-Sánchez and A. Ramos, Phys. Rev. E 92, 052313 (2015)

[35] J. E. Flores-Mena, P. García-Sánchez, and A. Ramos, Phys. Rev. E 99, 032603 (2019).

[36] A. Ramos, P. García-Sánchez, and H. Morgan, Curr. Opin. Colloid Interface Sci. 24, 79 (2016).

[37] P. García-Sánchez and A. Ramos, Langmuir 33, 8553 (2017).

[38] P. García-Sánchez, J. E. Flores-Mena, and A. Ramos, Micromachines 10, 100 (2019).

[39] G. Jeffery, Proc. Lond. Math. Soc. 14, 327 (1915).

[40] A. González, A. Ramos, N. G. Green, A. Castellanos, and H. Morgan, Phys. Rev. E 61, 4019 (2000).

[41] C. Zhao and C. Yang, Phys. Rev. E 83, 066304 (2011).

[42] A. Janotti and C. G. Van de Walle, Rep. Prog. Phys. 72, 126501 (2009).

[43] J. Goldberger, D. J. Sirbuly, M. Law, and P. Yang, J. Phys. Chem. B 109, 9 (2005). 
[44] M. Z. Bazant and T. M. Squires, Phys. Rev. Lett. 92, 066101 (2004).

[45] J. Lyklema, Fundamentals of Interface and Colloid Science (Academic Press Limited, London, 1995).

[46] C. Grosse and V. N. Shilov, J. Phys. Chem. 100, 1771 (1996).

[47] H. Jiang, J. Hu, F. Gu, and C. Li, Particuology 7, 225 (2009).

[48] C. G. Van de Walle, Phys. Rev. Lett. 85, 1012 (2000).
[49] M. Trejo, P. Santiago, H. Sobral, L. Rendón, and U. Pal, Crystal Growth Des. 9, 3024 (2009).

[50] R. Bhardwaj, A. Bharti, J. P. Singh, K. H. Chae, N. Goyal, and S. Gautam, Heliyon 4, e00594 (2018).

[51] S. Inguva, S. K. Marka, R. K. Vijayaraghavan, E. McGlynn, V. V. Srikanth, and J.-P. Mosnier, J. Phys. Chem. C 119, 4848 (2015). 